\section{Viktor-Ruppert- Medaille 1999}

Die im Jahr 1998 vom Ärzteverband Deutscher Allergologen (ÄDA) ins Leben gerufene Preisverleihung in Form der Viktor-Ruppert-Medaille eine Auszeichnung, die in Rückbesinnung auf die Ursprünge des Verbandes nach ihrem Gründer, dem Kölner Internisten und Allergologen, Viktor Ruppert, benannt ist wird im Jahre 1999 aufgrund eines einstimmigen Vorstandsbeschlusses des ÄDA für herausragende Verdienste um die Allergologie an Herrn Professor Dr. med. L. Jäger verliehen. Die Auszeichnung wird im Rahmen des Jenaer Colloquium Allergicum ,Allergie 2000 - Rückblick und Ausblick“" am 8. Mai 1999 übergeben. Der letztjährige Preisträger war Herr Professor Dr. rer. nat. Dieter Haustein.

gung der Allergiepatienten zur Folge. „Nach Untersuchungen staatlicher Gesundheitsämter ist jedes vierte Kind im Einschulungsalter manifest allergisch erkrankt. Jedes siebte Kind leidet sogar an allergischem Asthma bronchiale. Doch bisher erhielten aufgrund sorgfältiger Erhebungen lediglich $4 \%$ der Allergiepatienten eine qualifizierte Labordiagnostik und nur $7 \%$ eine qualifizierte Therapie“", so J. Wenning, Präsident des ÄDA.

„Die Budgetierung ärztlicher Leistungen hat in der Vergangenheit bereits dazu geführt, daß in mehreren Bereichen der Kassenärztlichen Vereini- gung nur bis zu 1,50 DM pro Patient für die allergologische Diagnostik pro Quartal zur Verfügung standen. Mit diesem Betrag waren nicht einmal die Kosten für einen einzigen AllergieHauttest gedeckt, wobei für eine qualifizierte Untersuchung 20 bis 40 Tests erforderlich wären. Um einem Patienten eine vollständige Diagnostik zukommen $\mathrm{zu}$ lassen, benötigen wir ein dreiviertel Jahr. Denn sonst würde der Kapitalrahmen eines Quartals gesprengt“, so Wenning.

Doch gerade bei Allergien ist die Früherkennung überaus wichtig, um Spätfolgen zu vermeiden. Studien zufolge erkranken rund $40 \%$ der Heuschnupfenpatienten nach 8 Jahren an Asthma. Asthma schränkt die Lebensqualität der Patienten erheblich ein und kann sogar lebensbedrohlich sein.

„Es darf nicht sein, daß die fortschreitende Zunahme von Allergien, die heute schon etwa 18 Millionen Menschen in der Bundesrepublik betreffen, aus Gründen einer kaum mehr nachvollziehbaren Budgetierung billigend in Kauf genommen wird“, erklärt Wenning.

\section{Eine Maßnahme: Vorstellen des Allergie-Weißbuches}

Die Situation macht rasches Handeln notwendig. Deshalb haben Dr. Wenning und Prof. Dr. Dr. Johannes Ring,

\title{
Neu: Fortbildungsreferat des ÄDA
}

In der Vorstandssitzung im Januar 1999 wurde auf Beschluß des Vorstandes ein eigenes Fortbildungsreferat des Ärzteverbandes Deutscher Allergologen eingerichtet. Mit der Leitung wurden Herr Professor Günther Forck, Kirchherrngasse 14, D-48143 Münster, Tel. 0251/42811, sowie Herr Professor Dieter Kleinhans, Kolpingstraße 2, D-70736 Fellbach, Tel. 0711/5181507 beauftragt.

Das Fortbildungsreferat ist unter anderem auch dazu gedacht, die Regionalverbände des Ärzteverbandes Deutscher Allergologen bei der Durchführung von Fortbildungsveranstaltungen in der Allergologie in Zusammenarbeit mit den kooperativen Mitgliedern des Verbandes zu unterstützen.

Präsident der DGAI (Deutsche Gesellschaft für Allergologie und klinische Immunologie), sich entschlossen, ein Allergie-Weißbuch mit allen wichtigen Daten zur Epidemiologie, Ökonomie, Diagnostik, Therapie und Prävention zu erstellen. Das Weißbuch richtet sich in erster Linie an die Gesundheitsministerien, Parlamentarier, KVen, Spitzenverbände der Krankenkassen und Jour- 